\title{
High resolution comparative genomic hybridisation analysis reveals imbalances in dyschromosomal patients with normal or apparently balanced conventional karyotypes
}

\author{
Maria Kirchhoff ${ }^{1}$, Hanne Rose ${ }^{1}$, Jan Maahr ${ }^{1}$, Tommy Gerdes ${ }^{1}$, Merete Bugge ${ }^{2}$, \\ Niels Tommerup ${ }^{2}$, Zeynep Tümer ${ }^{2}$, James Lespinasse ${ }^{3}$, Peter KA Jensen ${ }^{4}$, Jutta Wirth ${ }^{5}$ and \\ Claes Lundsteen ${ }^{1}$
}

\begin{abstract}
${ }^{1}$ Cytogenetic Laboratory, Department of Clinical Genetics, Juliane Marie Center, The National University Hospital, Copenhagen; ${ }^{2}$ Department of Medical Genetics, Institute of Medical Biochemistry and Genetics, Panum Institute, University of Copenhagen, Copenhagen, Denmark; ${ }^{3}$ Cytogenetics Laboratory, Central Hospital, Chambery, France; ${ }^{4}$ Department of Clinical Genetic, Århus kommunehospital, Århus, Denmark; ${ }^{5}$ Max-Planck Institute for Molecular Genetics, Berlin, Germany
\end{abstract}

\begin{abstract}
A sensitive technique is needed for screening whole genome imbalances in dyschromosomal patients when G-banding shows normal karyotypes or apparently balanced translocations. In this study we performed highly sensitive comparative genomic hybridisation analysis on a number of such cases and revealed chromosomal imbalances in all. European Journal of Human Genetics (2000) 8, 661-668.
\end{abstract}

Keywords: comparative genomic hybridisation; chromosome analysis; chromosome aberrations; dyschromosomal

\section{Introduction}

Chromosome aberrations are the most common known cause of mental and physical deficiencies. During the approximately 40 years that cytogenetic analysis has been available as a diagnostic tool a number of clinical syndromes have proved to be caused by chromosome anomalies (eg Down syndrome, Turner syndrome and Klinefelter syndrome). Banding techniques have made it possibleto reveal a number of small structural chromosome aberrations. However, since some small structural chromosome abnormalities may be overlooked by G-banding analysis, additional techniques for screening for small imbalances in dyschromosomal patients are needed.

Such techniques may also be of value for apparently balanced de novo translocations detected by prenatal diagnostics. Of these $6-10 \%$ are pathogenic ${ }^{1,2}$ and may actually be unbalanced, but they cannot be distinguished from true

Correspondence: Maria Kirchhoff, Cytogenetic Laboratory 4052 Rigshospitalet, Blegdamsvej 9, DK-2100 Copenhagen Ø Denmark. Tel: +4535454857; Fax: +45354542 57; E-mail: markir@rh.dk

Received 20 October 1999; revised 11 April 2000; accepted 2 May 2000 balanced translocations by conventional cytogenetic techniques.

Multiplex-FISH and spectral karyotyping are whole genome screening techniques that have been successfully used for cytogenetic diagnostics of constitutional chromosomal abnormalities in pre- and postnatal applications. ${ }^{3,4}$ However, small deletions and duplications are in danger of being undetected by these techniques. YAC mapping of cryptic deletions in apparently balanced translocations in dyschromosomal patients have likewise shown to be successful. ${ }^{5,6}$ This technique, however, is laborious and is not suitable for patients with normal G-banded karyotypes.

Comparative genomic hybridisation $(\mathrm{CGH})$ is a screening technique for whole genome imbalances. ${ }^{7}$ The sensitivity of $\mathrm{CGH}$ is usually considered to be relatively low; however, we developed the tech nique further in order to increase sensitivity as well as specificity. ${ }^{8,9}$ This has enabled us to detect deletions below $10 \mathrm{Mbp}$ with very high specificity (the smallest so far being $3 \mathrm{Mbp}) .{ }^{10}$ Thus the improved high resolution technique supplements the chromosome banding and FISH techniques mentioned above and it is probably especially advantageous for detecting small deletions and 
duplications. In this study high resolution CGH was applied to one embryonic tissue case and nine cases of dysch romosomal patients. The latter cases, along with a number of similar cases, were referred to our laboratory for $\mathrm{CGH}$ analysis because the phenotypes of the patients were characteristic for chromosomal imbalance, despite the fact that conventional G-banding showed normal karyotypes or apparently balanced translocations. The initial G-band analyses were either performed at our laboratory or at other cytogenetic laboratories. In contrast to the G-banding results, high resolution $\mathrm{CGH}$ analysis revealed chromosome imbalances in the 10 cases presented in this work.

We believe that the $\mathrm{CGH}$ technique will turn out to be an indispensable adjunct to conventional chromosome analysis.

\section{Materials and methods \\ Cases}

Case 1 was embryonic tissue. The cytogenetic abnormalities of this case were known prior to CGH analysis since a partial monosomy 18qter was accidentally found in a chorionic villus case by interphase FISH with a locus-specific probe for chromosome18qter. However, no abnormalities were detected on the G-banded karyotype. The father of the foetus was shown by FISH to have a balanced 11;18 translocation which was also undetectable by G-banding. The father had a dyschromosomal brother with normal conventional karyotype.

Cases 2-10 were all blood samples derived from dyschromosomal patients. The phenotypes of the patients showed dysmorphic figures with congenital malformation and/or mental retardation. The imbalances of these cases were all revealed by $\mathrm{CGH}$ analysis. All cases were previously karyotyped by conventional cytogenetics according to standard protocols.

Karyotypes by G-banding: cases 1-6: normal karyotypes. Case 7: balanced $\mathrm{t}(1 ; 4)$ de novo, $\mathrm{t}(3 ; 13)$ de novo. Case 8 : balanced $t(1,6,5)$ de novo. Case9: balanced $t(3 ; 5)$ de novo. Case 10: normal karyotype (see Table 1). Case 9 was obtained from Coriell Cell Repositories, Coriell Institute for Medical Research, Camden, NJ, USA (Repository number GM 10607).

Prior to CGH, FISH was used in Case 1 - Whole chromosomes painting probes for chromosome 11 (BRL - now Oncor, Gaithersburg, MD, USA) and 18 (Oncor, Gaithersburg, $M D, U S A)$ and a locus specific probe for chromosome18qter, (IG-18, obtained from former Integrated Genetics, Framingham, MA, USA).

After CGH, FISH was used in Case 1 - telomeric probes for chromosome11 (TL1102-15 (band 11q25)) and chromosome18 (TL1802-15 (band 18q23)) both obtained from AL Technologies, Arlington, VA, USA; Case 3 - YAC probes for the following loci: D2S2227, D2S123, D2S2251, D2S378, D2S370, D2S2198, D2S147, D2S380, D2S2293 (CEPH, Paris, France), loci in italics were found to be deleted; Case4 - YAC probe $885 \mathrm{~d} 10$ (CEPH); Case 7 - YAC probes for the following loci: D13S159, D13S277, D13S1267, D13S174, D13S259, D13S274, D13S173, 13WI-9114, 13WI-6500, D13S261 $(\mathrm{CEPH})$, loci in italics were found to be deleted; and Case 10-chromosome9 centromere specific probe (D9Z1, Oncor)).

Reference DNAs for CGH analysis were obtained from peripheral blood drawn from karyotypically normal males and females.

High molecular weight genomic DNA was prepared by extractions on Qiagen Genomic Tip column (Qiagen, Hilden, Germany), or Puregene DNA isolation kit (Puregene, Gentra Systems; Minneapolis, USA).

\section{CGH}

CGH was performed as described previously. ${ }^{8,9}$ Briefly, patient DNA and normal reference DNA were labelled with FITC-12-dUTP and Texas Red-5-dUTP (DuPont, Boston, MA, USA), respectively. Four hundred ng of each DNA and $20 \mu \mathrm{g}$ Cot1 DNA were hybridised to normal metaphase chromosomes. Slides were hybridised for 4 days, washed, and counterstained with 4,6-diamidino-2-phenylindole. CGH

Table 1 Summary of G-banding, CGH and FISH data

\begin{tabular}{|c|c|c|c|c|}
\hline $\begin{array}{l}\text { Case } \\
\text { no. }\end{array}$ & $\begin{array}{l}\text { G-banding } \\
\text { analysis }\end{array}$ & CGH analysis & $\begin{array}{l}\text { Confirmation } \\
\text { of CGH } \\
\text { results by } \\
\text { G-banding }\end{array}$ & $\begin{array}{l}\text { Conformation } \\
\text { of CGH } \\
\text { results by } \\
\text { FISH }\end{array}$ \\
\hline \multirow[t]{2}{*}{1} & \multirow[t]{2}{*}{$\begin{array}{l}46, X X \\
\text { de novo }\end{array}$} & $\begin{array}{l}\text { rev ish enh } \\
\text { (11q25) }\end{array}$ & no & yes \\
\hline & & $\begin{array}{l}\text { rev ish dim } \\
\text { (18q23) }\end{array}$ & no & yes \\
\hline 2 & $\begin{array}{l}46, X Y \\
\text { de novo }\end{array}$ & $\begin{array}{l}\text { rev ish dim } \\
(1 q 22)\end{array}$ & yes & n.d. \\
\hline 3 & $\begin{array}{l}46, X Y \\
\text { de novo }\end{array}$ & $\begin{array}{l}\text { rev ish dim } \\
\text { (2p15) }\end{array}$ & no & yes \\
\hline 4 & $\begin{array}{l}46, X Y \\
\text { de novo }\end{array}$ & $\begin{array}{l}\text { rev ish enh } \\
\text { (10q11) }\end{array}$ & no & yes \\
\hline 5 & $46, X X$ & $\begin{array}{l}\text { rev ish dim } \\
(2 q 37)\end{array}$ & yes & n.d. \\
\hline 6 & $46, X X$ & $\begin{array}{l}\text { rev ish dim } \\
\text { (7p15) }\end{array}$ & yes & n.d. \\
\hline 7 & $\begin{array}{l}46, X Y, t(1 ; 4) \\
(q 31 ; q 21.2) \\
\text { t(3;13)(p14.1; } \\
\text { q33) de novo }\end{array}$ & $\begin{array}{l}\text { rev ish dim } \\
\text { (13q33) }\end{array}$ & no & yes \\
\hline 8 & $\begin{array}{l}46, X X, t(1,6,5) \\
\text { (p13;q14;p13) } \\
\text { de novo }\end{array}$ & $\begin{array}{l}\text { rev ish dim } \\
\text { (6q14) }\end{array}$ & no & n.d. \\
\hline \multirow[t]{2}{*}{9} & $\begin{array}{l}46, X Y, t(3 ; 5) \\
(p 23 ; p 13)\end{array}$ & $\begin{array}{l}\text { rev ish dim } \\
(2 q 24)\end{array}$ & yes & n.d. \\
\hline & de novo & $\begin{array}{l}\text { rev ish dim } \\
(5 p 13)\end{array}$ & no & n.d. \\
\hline 10 & $46, X X$ & $\begin{array}{l}\text { rev ish enh } \\
\text { (9) }\end{array}$ & yes $^{a}$ & yes $^{b}$ \\
\hline
\end{tabular}

n.d.: not done; atrisomy 9 was detected in $4 \%$ of cells; ${ }^{b}$ trisomy 9 was detected in $46 \%$ of uncultured cells by interphase FISH. 
image capture was performed with a Cyto Vision (Applied Imaging, Sunderland, UK) interfaced to a DM RBE fluorescence microscope (Leica, Heerbrugg, Switzerland) and images were transferred to a Magiscan image analysis system (Applied Imaging, Sunderland, UK). In each case, 10 metaphases were analysed. Detection of aberrations was performed by standard reference intervals as described in Kirchhoff et al. ${ }^{9}$

Briefly, along the mean ratio profiles the $99.5 \%$ confidence interval of each mean ratio profile value was compared with a corresponding $99.5 \%$ standard reference interval based on an average of 17 normal cases. The standard reference interval is especially wide at profile areas where CGH measurements are known to be unreliable. Whenever no overlap existed between the two intervals, the corresponding chromosome region was designated 'aberrant'. The standard reference interval was scaled automatically to fit the individual test case.

\section{Results}

Table 1 summarises the results of the G-banding analysis and the $\mathrm{CGH}$ analysis and states whether or not the $\mathrm{CGH}$ results could be confirmed by reinspection of the G-banded karyotype or by FISH. Chromosome aberrations were designated to bands according to the DAPI banding patterns. Figure 1 shows the $\mathrm{CGH}$ profiles and G-banding of the relevant chromosomes of all analyses. The following results were obtained.

Case 1: CGH analysis showed gain of chromosome11q25 and loss of chromosome18q23. Reinspection of the G-banded karyotype showed no imbalances. Whole chromosomes painting probes for chromosomes 11 and 18 showed that the duplicated part of chromosome 11 was translocated to the truncated chromosome18 (Figure2, A). Interphase FISH with a chromosome18qter locus specific probe showed only one signal from chromosome18 (Figure2, B). A telomere probe for chromosomal band $11 q 25$ showed that the translocation breakpoint of chromosome 11 was within this band (Figure2, C). A telomere probe for chromosome band 18q23 showed that the translocation breakpoint of chromosome18 was within this band (Figure2, D).

Case2: CGH analysis showed loss of chromosome1q22 and this finding was confirmed by reinspection of the G-banded karyotype (Figure1).

Case 3: CGH analysis showed loss of chromosome2p15. This was not evident by reinspection of the G-banded karyotype but FISH studies with YAC probes confirmed the deletion, which was mapped to be $4-5 \mathrm{Mbp}$ (Figure3, A, B and $C$ ).

Case 4: CGH analysis showed a gain of chromosome10q11. The gain was not confirmed by reinspection of the G-banded karyotype; however, FISH analysis with a chromosome10 locus specific probe showed increased or two signals on one of the chromosomes 10 (Figure3, D and E).
Case 5: CGH analysis showed loss of chromosome2q37 and this finding was confirmed by reinspection of the G-banded karyotype (Figure 1).

Case6: CGH analysis showed loss of chromosome7p15 and this finding was confirmed by reinspection of the G-banded karyotype (Figure 1).

Case 7: CGH analysis showed loss of chromosome 13q33. This deletion was located in the breakpoint of the chromosome 13 involved in the 3;13 de novo translocation of this case. This was not evident by reinspection of the G-banded karyotype, however FISH with YAC probes confirmed the deletion, which was mapped to be approximately $5 \mathrm{Mbp}$ (Figure 3, F, G and $\mathrm{H}$ ).

Case 8: CGH results showed a deletion of chromosome6q14. The deletion was not evident by reinspection of the G-banded karyotype and FISH studies have not been possible to carry out due to lack of sample material. The deletion of $6 q 14$ was located in the breakpoint of the chromosome 6 involved in the 1;6;5 de novo translocation of this case.

Case 9: CGH analysis showed deletions of chromosomes $2 q 24$ and 5p13. Only the deletion on chromosome 2 was confirmed by reinspection of the G-banded karyotype (Figure 1) and FISH studies could not be carried out due to lack of sample material. The deletion of $5 \mathrm{p} 13$ was located in the breakpoint of the chromosome 5 involved in the 3;5 de novo translocation of this case.

Case 10: CGH results showed gain of chromosome9. Screening of $100 \mathrm{G}$-banded metaphases showed trisomy 9 in 4 cells (chromosomes 9 from one of the trisomic cells is shown in Figure 1). Interphase FISH with a chromosome9 centromere specific probe performed on uncultured cells showed three signals in 46 of 100 counted cells (not shown).

No other aberrations were detected by CGH in any of the cases apart from the ones listed in Table 1 and no chromosome regions were excluded from analysis. The chromosomes of the parents were analysed with G-banding analysis and/or $\mathrm{CGH}$ whenever blood samples were obtainable. The analyses all showed that the chromosome aberrations found in the dyschromosomal children were de novo (see Table1).

\section{Discussion}

The cases in this study (except for case 1 ) were analysed by CGH due to suspicion of chromosomal imbalance despite the fact that conventional G-banding showed normal karyotypes or apparently balanced translocations. The $\mathrm{CGH}$ analyses showed abnormalities in all cases. However, since the experience with high resolution $\mathrm{CGH}$ was limited, it was desirable to confirm the findings by other methods. Ten of the 12 imbalances found by CGH have been confirmed by either FISH or reinspection of the G-banded karyotype or both. It could not be excluded that the two unconfirmed deletions (Cases 8 and 9) were false positive results. However, as previously shown ${ }^{9}$ false positive results are practical ly non- 
existing at the level of confidence used in the $\mathrm{CGH}$ analyses of this work. Moreover, the reliability of the two deletions found in Cases 8 and 9 is strengthened by the fact that the deletions were found at the breakpoints of chromosomes involved in apparently balanced de novo translocations. Furthermore, the patient with the deletion on chromoso- me5p (Case9) was clinically suspected of cri-du-chat syndrome.

The success of CGH analysis in detecting imbal ances in this study can be related to a number of factors. The sensitivity of the $\mathrm{CGH}$ technique seems higher than that of G-banding at the 300-600 band level, since for some Cases $(1,3,4,7)$,
11

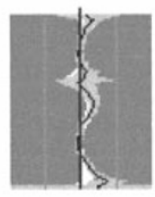

Case 1

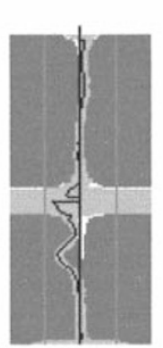

Case 2

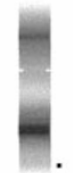

1

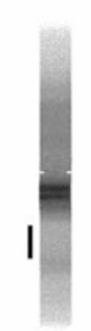

2

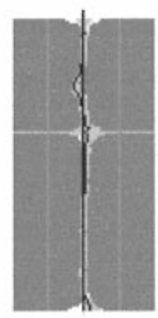

Case 3

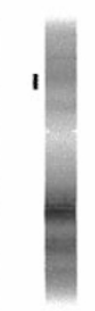

10

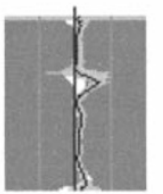

Case 4

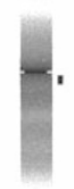

Case 5

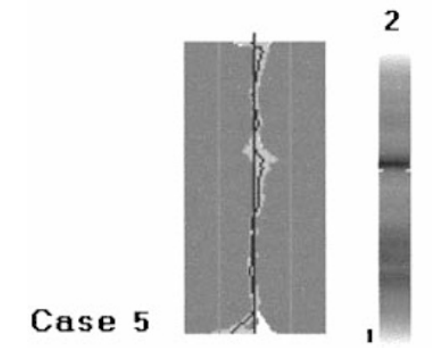

2

Figure 1 Continued on next page.
11

18

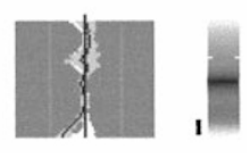

1

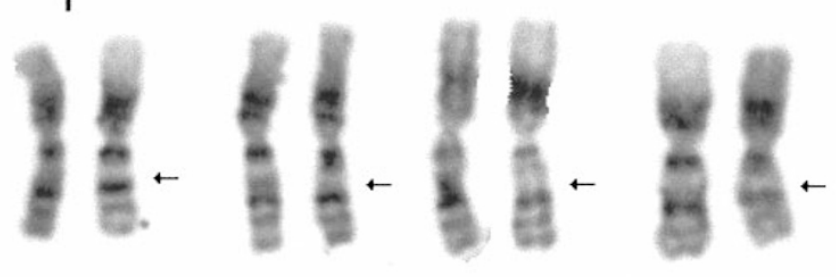

2

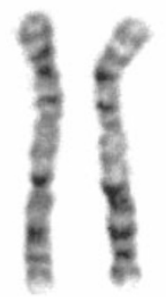

10

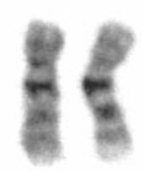

2

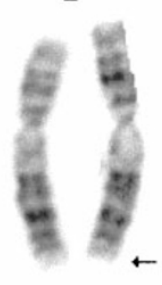

18
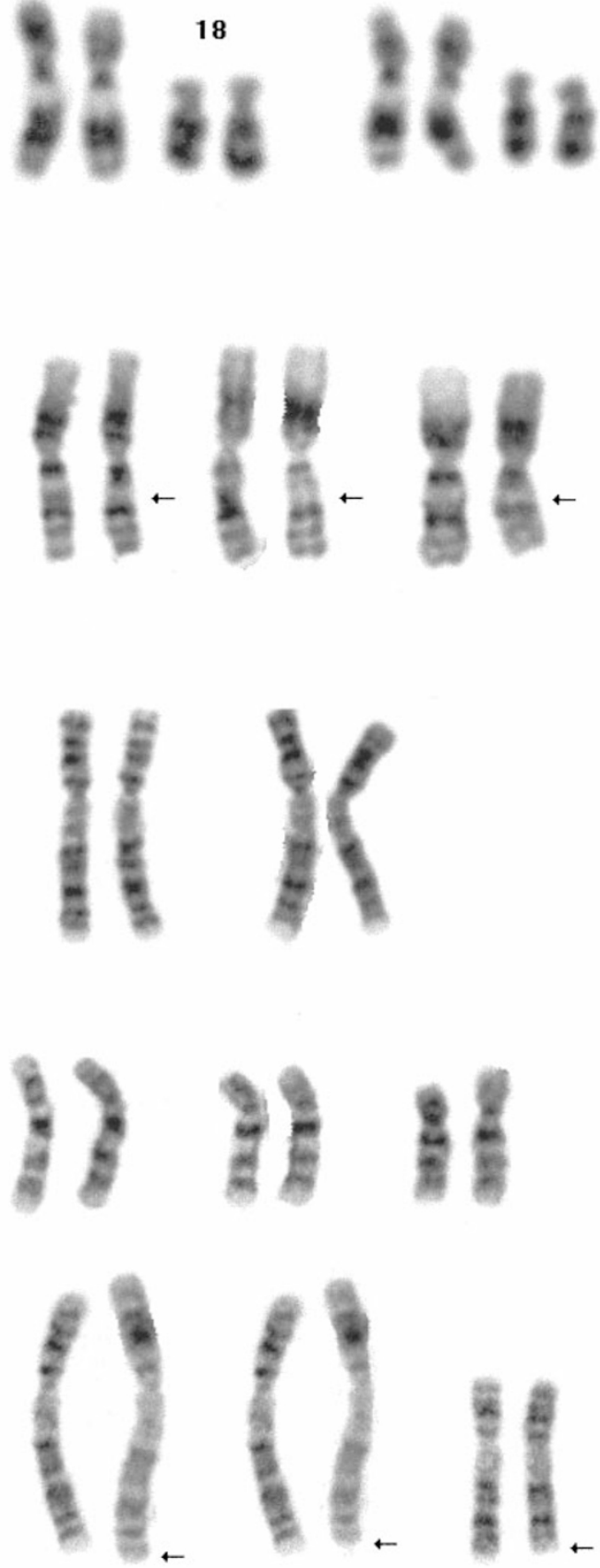
Case 6
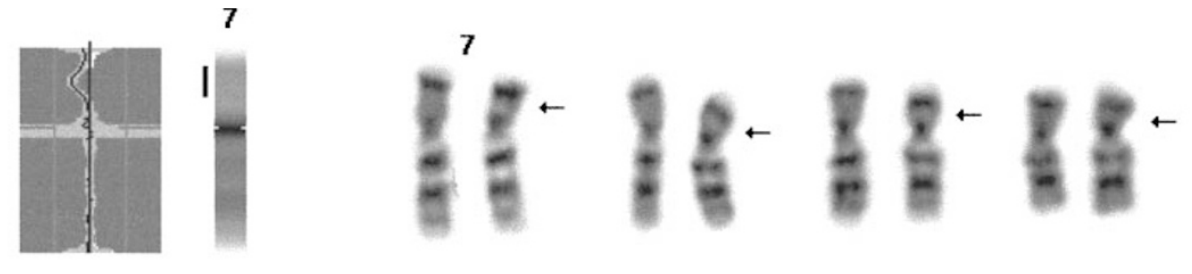

13

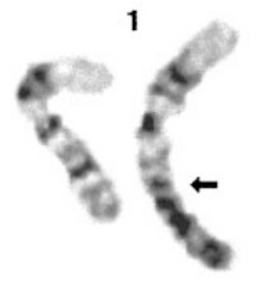

4

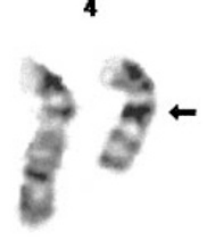

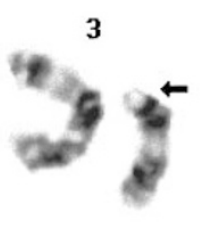

6

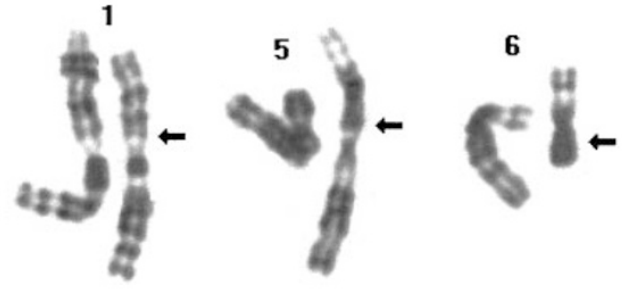

2

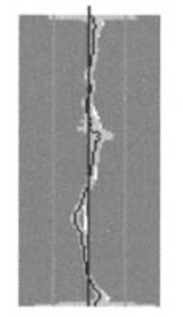

Case 9
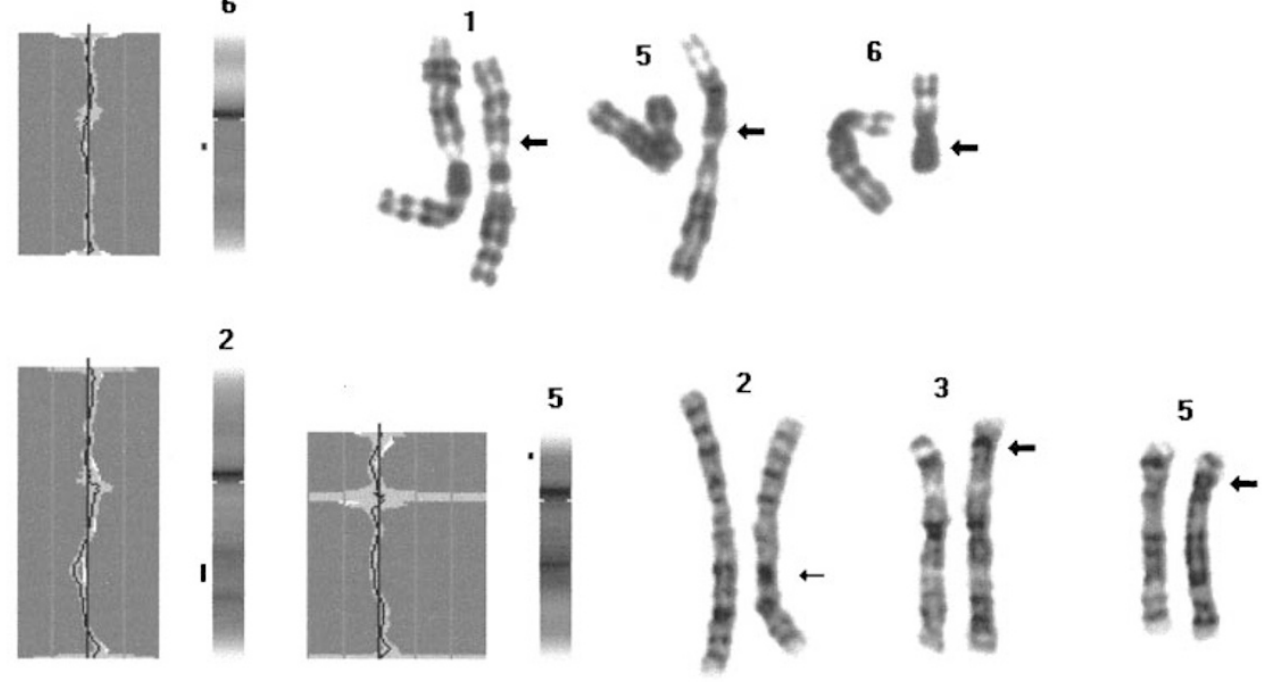

\section{Case 10}
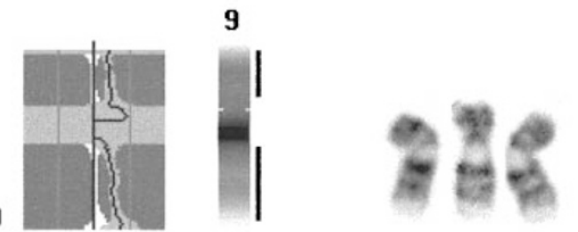

Figure $1 \mathrm{CGH}$ results and G-banding analysis of selected chromosomes of all 10 cases. Mean ratio-profiles and $99.5 \%$ confidence intervals (grey) of the cases are shown. 99.5\% standard reference intervals (white) are positioned underneath the confidence intervals. Aberrations are detected whenever the two intervals do not overlap. Vertical lines represent ratio 0.5, 1.0 and 1.5. Gains and losses are shown as black bars positioned respectively to right and left of the mean inverted DAPI banded pattern. Bold arrows indicate breakpoints in translocations and light arrows indicate chromosome aberrations confirmed by reinspection of the G-banded karyotype.

confirmed by FISH, it was not possible subsequently to confirm the $\mathrm{CGH}$ findings by reinspection of the G-banded karyotype. We know from a previous study that detection of deletions of $3 \mathrm{Mbp}$ is feasible by high resolution $\mathrm{CGH}^{10}$ and we have previously detected a number of Prader Willi/ Angelman deletions by $\mathrm{CGH}$ (see Kirchhoff et al, ${ }^{9}$ for example). These deletions are usually considered to be approximately $4 \mathrm{Mbp}^{11}$ and are thus likely to be undetected by normal G-banding at the 300-600 band level.
For some of the cases reinspection of the G-banded karyotype confirmed the CGH findings and for two Cases (2 and 6) the abnormalities were actually quite conspicuous once it was decided on where to look. This clearly illustrates a weakness of the G-banding technique in comparison to CGH. G-band analysis is dependent on the qualifications and the actual performance of the cytogeneticist and this makes the technique somewhat subjective. Even highly skilled cytogeneticists may overlook small imbalances if no prior 


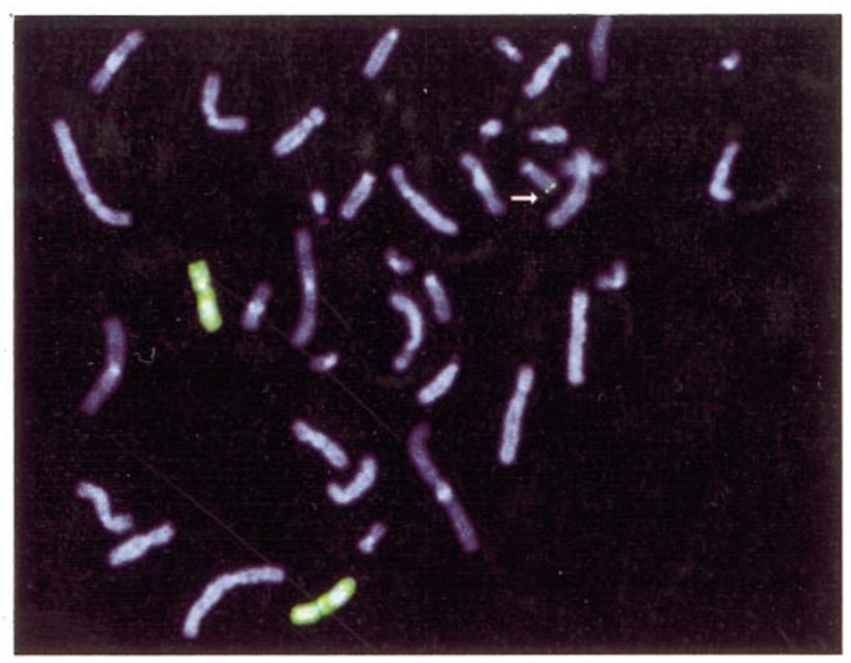

A

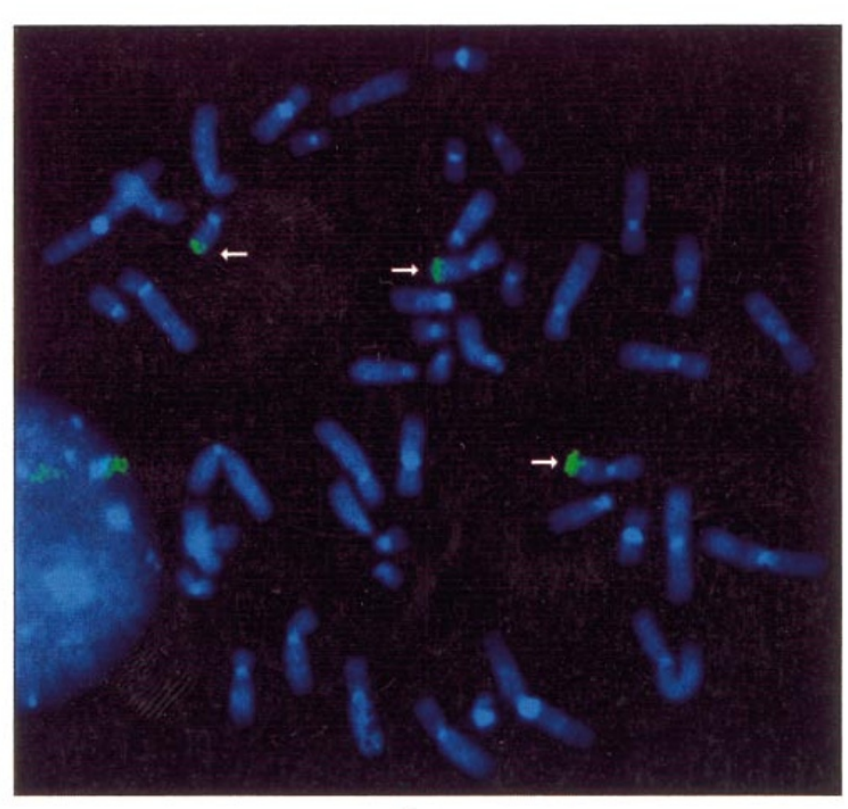

C

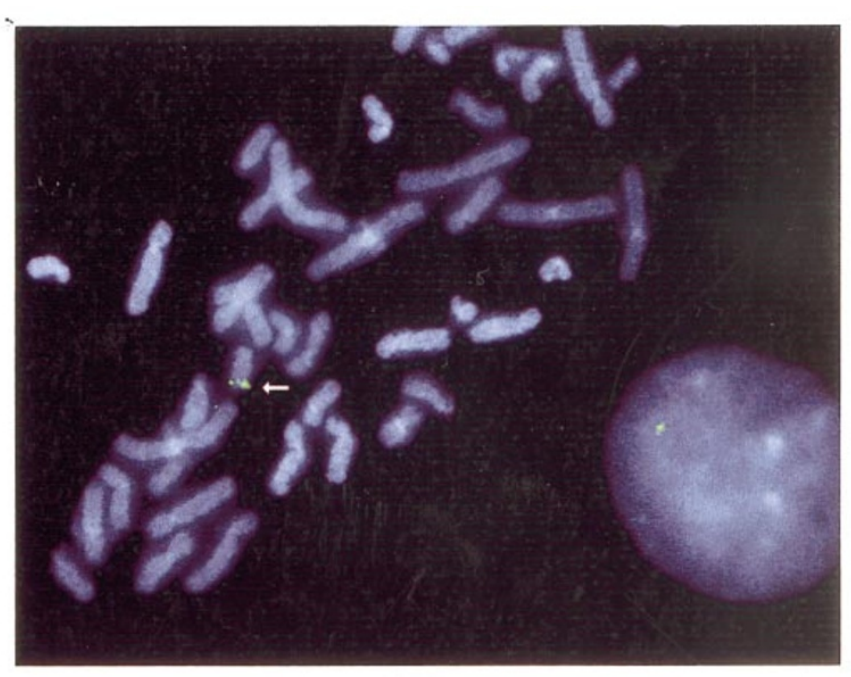

B

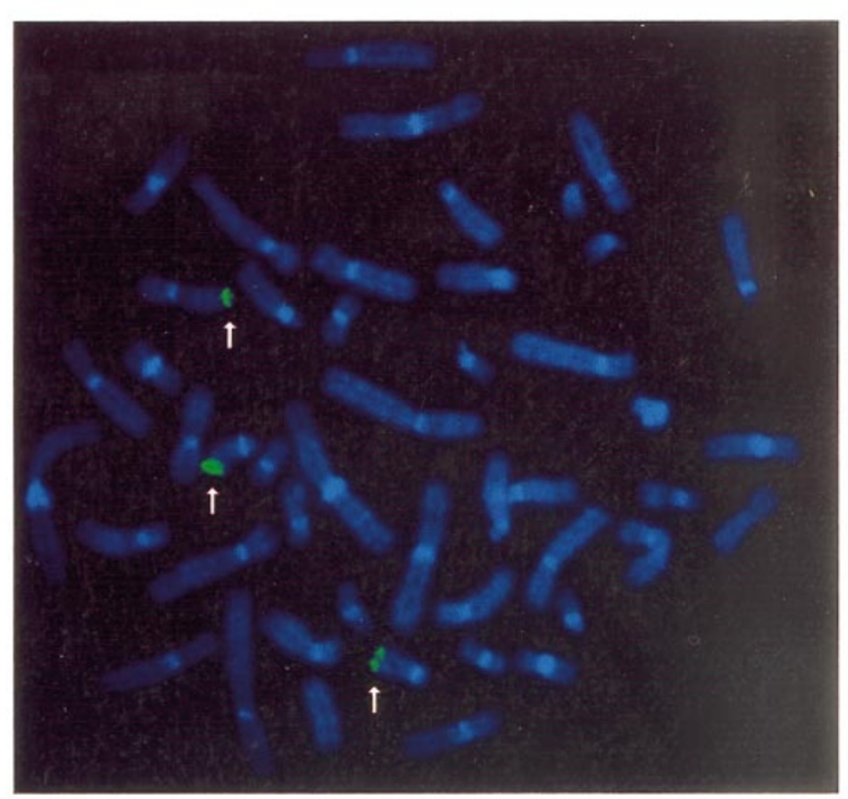

D

Figure 2 FISH analyses of case1: A Whole chromosome painting probe for chromosome 11 shows chromosome 11 material at the distal end of chromosome 18q. B Locus specific probe for chromosome 18qter shows signal from only one chromosome 18. C Telomere probe for chromosome band 11q25 shows that the translocation breakpoint is within this band. D Telomere probe for chromosome band 18q23 shows that the translocation breakpoint is within this band.

knowledge of the abnormality is available, and as to the cases carrying the translocations, it may be impossible to detect small imbalances since exchange of chromosomal material may alter the appearance of the bands at the breakpoints making it difficult to detect deletions.
It is possible that the finding of a translocation in a G-banded karyotype may distract the cytogeneticists attention from detecting other abnormalities. This might have been so when Case 9 was karyotyped. A chromosome3;5 translocation was detected in this case but an interstitial 


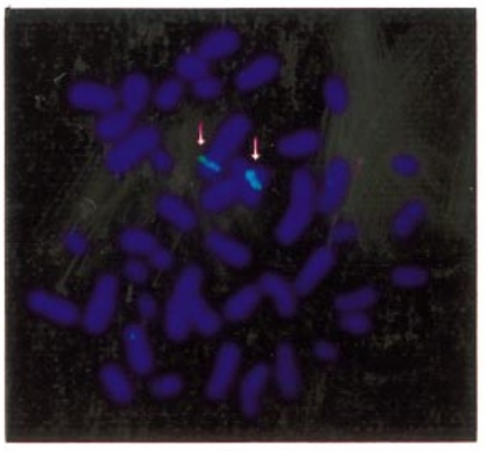

A

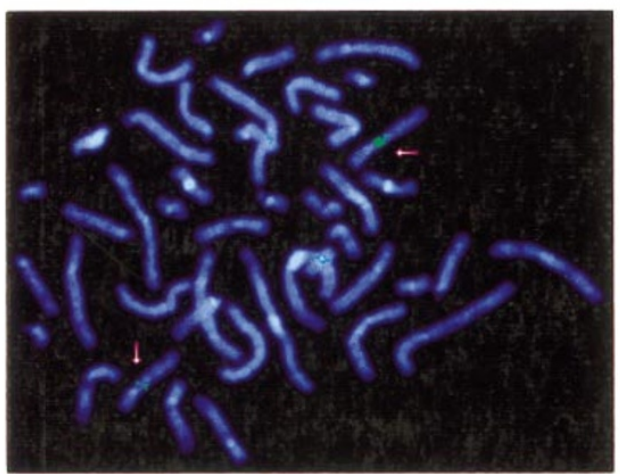

D

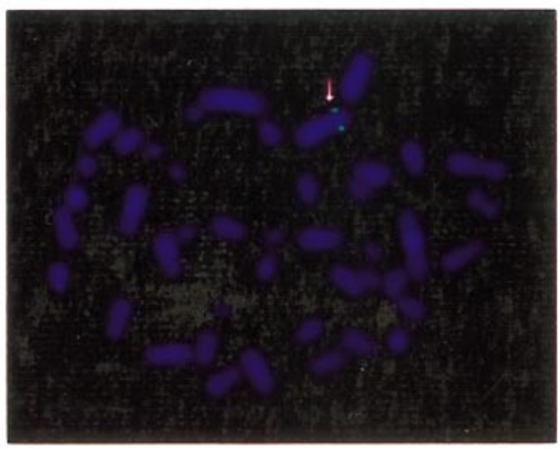

B

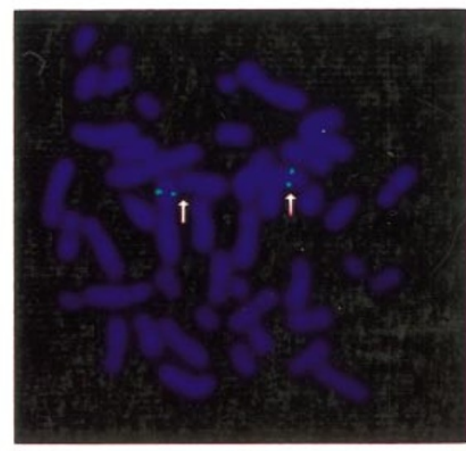

C

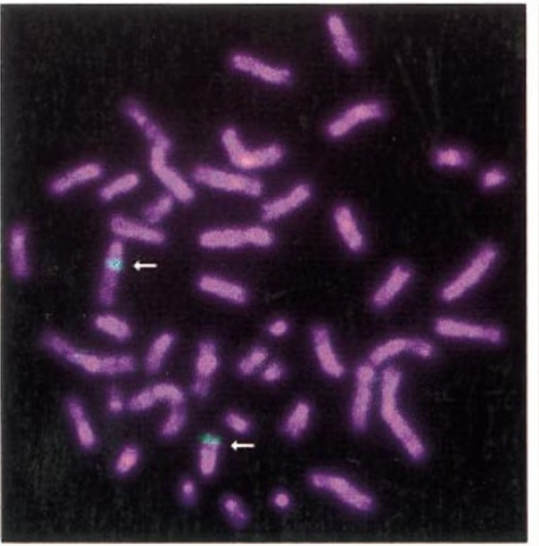

$\mathbf{F}$

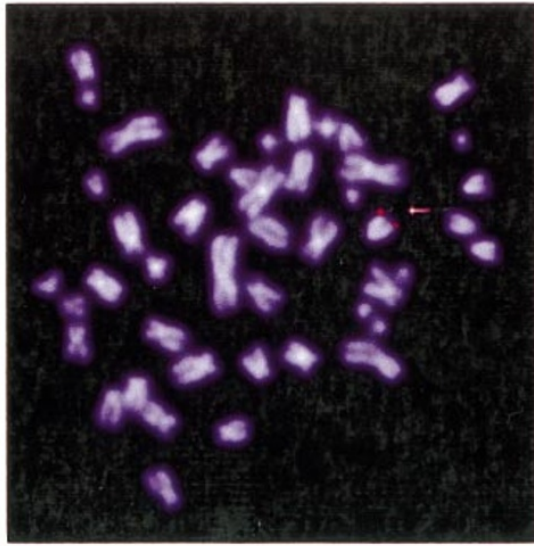

G

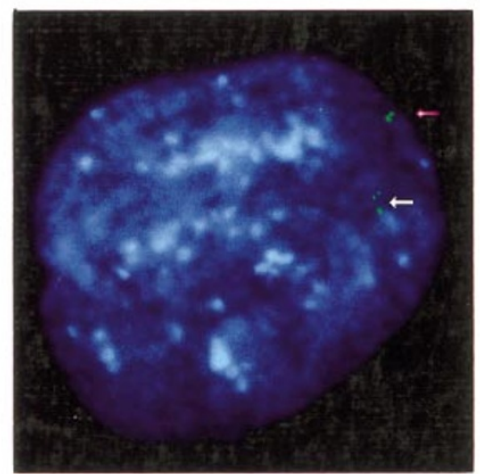

E

Figure 3 Fish analyses of Cases 2, 4 and 7: A Case 3. Chromosome2 YAC probe for locus D2S123 shows signals from both chromosomes2. The locus is proximal to the deletion. B Case 3. Chromosome2 YAC probe for locus D2S370 shows signal from the normal chromosome2. C Case 3. Chromosome2 YAC probe for locus D2S147 shows signals from both chromosomes2. The locus is distal to the deletion. D Case4. Chromosome 10 YAC probe $885 \mathrm{~d} 10$ shows enhanced signal from the chromosome 10 with the duplication. E Case4. Chromosome 10 YAC probe $885 \mathrm{~d} 10$ shows distinct signals from the chromosome 10 with the duplication. F Case 7. Chromosome 13 YAC probe for locus D13S277 shows signal from the normal chromosome 13 and the derivative chromosome13. The locus is proximal to the deletion. G Case 7. Chromosome13 YAC probe for locus D13S259 shows only signal from the normal chromosome13. H Case7. Chromosome13 YAC probe for locus D13S173 shows signal from the normal chromosome13 and from the derivative chromosome3. The locus is distal to the deletion. 
deletion on chromosome2q was overlooked. This deletion was, however, confirmed by G-banding after CGH analysis.

Variability of the quality of G-banded chromosomes is an additional problem that is experienced by all laboratories working with the technique and it is also apparent in Figure1. The G-banded chromosomes shown in this figure are derived from four different laboratories.

$\mathrm{CGH}$ analysis is characterised by a higher degree of objectivity than G-banding analysis. The metaphases used for the analysis are chosen by the individual performing the analysis, but the final detection of abnormalities is performed by statistical analysis. This objectivity paired with high sensitivity and specificity makes CGH analysis an excellent choice for detection for chromosomal aberrations in dyschromosomal patients when conventional G-banding shows normal chromosomes or apparently balanced translocations.

Case 10 exemplifies a different problem from the rest of the cases since the abnormality in it was not small. However, the trisomy 9 mosaic almost entirely disappeared during cultivation. CGH has gained great popularity in cancer research and one of the reasons for this is that clonal selection due to cultivation is avoided. It may be that clonal selection during cultivation of abnormal mosaic blood cases is a more widespread phenomenon than previously suspected.

It is still unclear if the aberrations found in the cases of this study are responsible for the clinical presentations of the patients. Loss of chromosomal material is usually associated with a seriously affected phenotype. Nevertheless, it cannot be ruled out that some findings may be of no apparent clinical significance. The small duplication on chromosome arm 10q in Case4 may bea chromosomal variation present in some normal individuals. A likewise small duplication at $15 q$ has been described in normal individuals. ${ }^{12}$ Since conventional banding techniques do not detect small imbalances, the extension of such chromosomal variations in normal populations is unknown, and it is possible that several types of euchromatic variants may be transmitted in families, without reproductive or clinical effect.

It is of crucial importance to be able to diagnose dyschromosomal children. These children often go through a number of examinations with normal results, and without a diagnosis the parents are left in a very difficult situation in case of a new pregnancy. The use of CGH as an objective and sensitive screening technique for revealing chromosomal imbalances in dyschromosomal patients with normal or apparently balanced G-banded karyotypes seems promising. However, the $\mathrm{CGH}$ technique is both laborious and expensive to perform and economy may be a limiting factor for routine use. The crucial question is how often will imbal- ances be detected in dyschromosomal patients by $\mathrm{CGH}$. We do not know the answer to this question since the imbalances presented in this work were found among a limited number of cases which were not referred to us on an organised basis.

In order to address the question and further characterise the potential of the technique for this specific clinical application we are performing a larger prospective study of dyschromosomal patients with either normal karyotypes or apparently balanced de novo translocations referred to our laboratory from paediatric departments all over Denmark.

\section{Acknowledgements}

This work was supported by the Ivan Nielsens Fond for personer med specielle sindslidelser.

\section{References}

1 Wassman ER, Cheyovich DL, Nakahara Y: 'Possibly' de novo translocations: prenatal risk counseling. Am J Obstet Gynecol 1989; 161: 698-702.

2 Warburton D: De novo balanced chromosome rearrangements and extra marker chromosomes identified at prenatal diagnosis: clinical significance and distribution of breakpoints. Am J Hum Genet 1991; 49: 995-1013.

3 Uhrig S, Schuffenhauer S, Fauth C et al: Multiplex-FISH for preand postnatal diagnostic applications. Am J Hum Genet 1999; 65: 448-462.

4 Schröck E, Veldman T, Padilla-Nash $\mathrm{H}$ et al: Spectral karyotyping refines cytogenetic diagnostics of constitutional chromosomal abnormalities. Hum Genet 1997; 101: 255-262.

5 Kumar A, Becker LA, Depinet TW et al: Molecular characterization and delineation of subtle deletions in de novo 'balanced' chromosomal rearrangements. Hum Genet 1998; 103: 173-178.

6 Wirth J, Nothwang HG, van der Maarel S et al: Systematic characterisation of disease associated balanced chromosome rearrangements by FISH: cytogenetically anchored YACs identify microdeletions and candidate regions for mental retardation genes. Med Genet 1999; 35: 271-278.

7 Kallioniemi A, Kallioniemi OP, Sudar D et al: Comparative genomic hybridization for molecular cytogenetic analysis of solid tumors. Science 1992; 258: 818-821.

8 Kirchhoff M, Gerdes T, Maahr J, Rose H, Lundsteen C: Automatic correction of the interfering effect of unsuppressed interspersed repetitive sequences in comparative genomic hybridization analysis. Cytometry 1997; 28: 130-134.

9 Kirchhoff M, Gerdes T, Rose H, Maahr J, Ottesen AM, Lundsteen C: Detection of chromosomal gains and losses in comparative genomic hybridization analysis based on standard reference intervals. Cytometry 1998; 31: 163-173.

10 Kirchhoff M, Gerdes T, Maahr J et al: Deletions below 10 megabasepairs are detected in comparative genomic hybridization by standard reference intervals. Genes Chrom Cancer 1999; 25: 410-413.

11 Christian SL, Bhatt NK, Martin SA et al: Integrated YAC contig map of the Prader-Willi/Angelman region on chromosome15q11-q13 with an average STS spacing of $35 \mathrm{~kb}$. Genome Res 1998; 8: 146-157.

12 Browne CE, Dennis NR, Maher E et al: Inherited interstitial duplications of proximal 15q: genotype-phenotype correlations. Am J Hum Genet 1997; 61: 1342-1352. 